\title{
Effect of Different Nursing Educational Methods about Correct use of Inhaler Devices on Clinical Improvement for Asthmatic Patients
}

\author{
Marwa Ali Almasry ${ }^{1}$, Neama Mamdouh Mostafa ${ }^{2}$, Saieda Abd Elhameed AbdElaziz ${ }^{3}$ \& Hanan Abdallah Abozeid ${ }^{4}$ \\ 1. Lecturer of Medical-Surgical Nursing Department, Faculty of Nursing, Assiut University, Egypt \\ ${ }^{2 .}$ Lecturer of Medical-Surgical Nursing Department, Faculty of Nursing, Assiut University, Egypt \\ 3. Assistant professor of Gerontological Nursing Department, Faculty of Nursing, Assiut University, Egypt \\ 4. Assistant professor of Gerontological Nursing Department, Faculty of Nursing, Assiut University, Egypt
}

\begin{abstract}
:
Background: Most asthmatic patients use their inhalers incorrectly; As a result, they are more vulnerable to poor clinical control and exacerbation. The study aim: was to evaluate the effect of different nursing educational methods (video, educational booklet, both booklet \& video) about correct use of inhaler devices on the clinical improvement for asthmatic patients. Methods: Design of the study: Quiz-experimental design. Setting: chest department and chest diseases outpatient clinics at Assiut University Hospitals Sample: 80 asthmatic patients were divided into four groups, 20 patients for each. Tools: Tool I: patient assessment sheet, Tool II: Global Initiative for Asthma scale (GINA), 2019, and Tool III: nursing educational methods. Results: According to the Global Initiative for Asthma scale, in excess of half of the patients $(60.0 \%)$ in the routine nursing instructions, booklet, and video groups had partially asthma control, while the majority of patients $(80.0 \%)$ in the video and booklet groups had well asthma control. Conclusions: The group of patients who received the video and the booklet had a statistically significant effect on improving the level of asthma control. Recommendation: All asthma patients should be given written and visual information about how to use inhalers devices correctly to minimize the complications arising from the incorrect use of inhalers.
\end{abstract}

\section{Keywords: Asthmatic patients, Clinical improvement, Inhaler devices \& Nursing educational methods}

\section{Introduction:}

Asthma affected an estimated 262 million people in 2019 and caused 461000 deaths (world health organization, 2019). Asthma is a common chronic disease that affects people of all ages and from all parts of the world around the globe. It causes a substantial burden of disease in people of all ages, including early death and impaired quality of life. Asthma affects 300 million individuals of all ages and ethnicities worldwide, with 1 in every 250 people dying from the disease. It is expected that by 2025 , another 100 million people would be affected. (The Global Asthma Report, 2018).

The most effective treatment option for asthma is inhalation therapy. Good inhaler technique is essential to get the most out of these medications and improve outcomes for the patient. The numerous types of inhalers on the market have varying specifications and need different approaches, resulting in a number of performance faults and a reduction in treatment efficacy. Up to 76 percent of patients make some form of an inhaler technique error (Miguel et al, 2019). Growing research suggests that instructional interventions and technique reviews help to control the disease (David et al, 2019).
Inhalation medications are the cornerstone of asthma therapy, but they can only be effective if they are used properly. When an inhaler is used correctly, the drug is delivered to the lungs, where it can help control asthma symptoms. According to the studies, incorrect inhaler technique can lead to inadequate asthma control and poor clinical consequences. Inhaler technique errors are still common among asthma patients, with no improvement in recent years (Chrystyn et al, 2017).

Using an inhaler incorrectly means that little or no medicine reaches the lungs. This can be due to a variety of factors such as the inhaler not being shaken before use, failing to properly position the inhaler, failure of the patient to hold their breath after inhalation, failure to breathe properly in and out, and failure to rinse their mouth with warm water after medication administration. The Global Initiative for Asthma published a study in 2016 urging nurses and clinicians to monitor for incorrect inhaler use and provide proper education (Global initiative for asthma, 2020)

Also the elderly patient with asthma may have concerns about the side-effects of the medication, poor inhaler technique or insufficient inspiratory flows, and asthma management can be affected by 
memory impairment or economic difficulties. (Hanania et al., 2011).

Because proper technique varies depending on the type of inhaler, patients must learn how to use their specific inhaler. Asthma control can be improved with simple verbal instructions and practical demonstrations of correct inhaler use. Written instructions alone about how to use the inhaler are insufficient. When patients are being prescribed inhalants for the first time, they should be taught how to use an inhaler by using other methods such as video chatting can be benefit, as well as checking their technique at subsequent consultations (Schantz et al., 2018)

Persistent assessment and reinforcement of the correct inhaler technique can help Asthma patients to get better medicine intake and improved treatment outcome. To help new asthma patients better understand the proper way to use an inhaler, as well as to provide some handy tips for experienced users, Patient education plays one of the most important roles in the patients' use and misuse of asthma inhalers (Global initiative for asthma ,2020). Inhaler misuse is comdmon, and the expenses are considerable, including more doctor visits, hospitalizations, and higher inhaler medicine use. All of these can be decreased by spending more time to teaching patients how to correctly use their prescribed inhaler device (Schantz et al., 2018).

Inhalation therapy is the most common treatment for asthma. When it comes to the elderly, the patients' capacity to use inhalers may be crucial. Elderly asthmatics, on the other hand, have been shown to improve their inhaler technique when given practical demonstration and instruction (Crane, et al., 2014)

To counteract for the increasing costs, new and effective methods to improve inhaler technique and ensure safe use of asthma inhalers are required. Current approaches for providing inhaler training include written instructions, graphics, video demonstrations, as well as individual and small group demonstrations (Lavorini, 2013)

\section{Significance of the study:}

Prevalence of asthma in Egypt was 6.7\% (Tarraf et al., 2018). Incorrect inhaling method can limit medicine deposition in the lungs, resulting in lower efficacy. Poor technique was estimated to be present in 14-90 percent (with an average of 50 percent) of cases in a study of 21 research looking at misuse of metered dosage inhalers (Giraud \& Roche, 2017).

In a systematic review of educational programs for adult self-management of asthma, education was linked to improved lung function, decrease numbers of days with restricted activities and fewer the rate of emergency departments visits (Guevara et a.l., 2018).

Aims of the study:

Was evaluate the effect of different nursing educational methods (video, educational booklet, both booklet \& video) about correct use of inhaler devices on the clinical improvement for asthmatic patients.

Hypothesis: To achieve the study's goal, the following research hypothesis was formulated:

1.Patients who receive video will have a higher mean score on the asthma symptoms control measure than patients who receives routine nursing instructions

2.Patients who receive educational booklets will have a higher mean score on the asthma symptoms control scale than patients who routine nursing instructions.

3.Patients who receive both the video and the booklet will have a higher mean score in the asthma symptoms control scale than those who receive only routine care.

Operational definition:

Asthma control: is the extent to which the manifestations of asthma can be observed in the patient, or have been reduced or removed by treatment.

Methods:

Research design: In this study, Quiz-experimental research design was used.

\section{Study variables}

The educational nursing methods were the independent variable in this study (routine nursing instructions, an educational booklet, clinical correct use of inhaler on video and both booklet \& video). The level of asthma symptom control was the dependent variable.

Setting:

The study was conducted at the chest department and chest diseases outpatient clinics at Assiut University Hospital.

Patients:

A purposive sample 80 asthmatic patients were divided into four groups of 20 for each group. The first group (control group) received routine nursing instructions; the second group received merely a nursing educational booklet; the third group was simply shown how to use an inhaler correctly through video only, and the fourth group received both the video and the booklet. To ensure randomization, the group receiving routine nursing instructions and clinical correct use of inhalers via video only received odd numbers, while a nursing educational booklet group only received even numbers, and the group of nursing educational booklet and video together received even numbers. 


\section{Inclusion criteria}

The study group was chosen based on the following criteria: Patients diagnosed with asthma and who use one of the following inhaler device (pressurized Metered Dose Inhaler (pMDI) with or without Spacer, Dry Powder Inhaler or Soft Mist), aged ranged from 18 to 65 years and those who visit the outpatient clinics regularly to take the monthly treatment.

\section{Sample size}

The power analysis to estimate the sample size was performed based on the result of previous study. Assuming power of $0.80 \%$ and 0.05 (one sided equivalence test). A total sample size of 50 participants is required the eligible patients were invited to participate after the assessment whether they meet all of the inclusion criteria $n=80$

\section{Tools of the study:}

Tool I: Patients Assessment sheet:

Part (1): Demographic data: including the patients' name, age, sex, residence, marital status, educational level, and occupation.

Part (2): Medical data: Previous admission to hospital, Number of admission in the last year, Age of start asthma, Patient's condition at discharge, Presence of cough, If present how much you cough, shortness of breath, wheezing, chest tightness or pain, and chronic diseases

Part (3): Risk factors for asthma exacerbations: This part contains 6 risk factors for asthma exacerbation, which included (Uncontrolled asthma symptoms, medications; high (SABA) short acting beta agonist use if $>1 * 200$ dose canister /month, inadequate ICS, poor adherence, Incorrect inhaler technique, comorbidities, and exposure, context)

Part (4): Complications of asthma: This included (pneumonia, respiratory infection, respiratory failure, status asthmatics, asthma exacerbation, lack of physical activity, fatigue, and sleep difficulties)

Tool II: The Global Initiative for Asthma (GINA) scale in adult , 2019:

This scale used to assess the level of asthma symptoms control in the past 4 weeks, It developed by the Global Initiative for Asthma (GINA) was established in 1993 in collaboration with the National Heart, Lung and Blood Institute and the World Health Organization, under the leadership of Drs Suzanne Hurd and Claude Lenfant,. It consists of four clinical parameters rated on two responses; one grade was awarded for the yes and zero to the no answer. The numerical results provide a score that represents an estimate of the level of asthma symptoms.

Scoring system, the total score can be categorized into 3 categories:

Well control asthma $=$ the patients don't have any of these clinical parameters
Partly control asthma $=$ if the patient has one to two of these clinical parameters

Uncontrolled asthma =when the patient complains from the presence of three to four clinical parameters.

Tool III: Correct inhaler use educational methods: It included three parts:

Part I: Nursing educational booklet: the instructions were developed by the researchers through reviewing of related literature. The instructions involved the knowledge about the correct technique inhaler device. This process included four parts: 1) the researcher interview each patient to provide a step-by-step explanation on how to use the inhaler device correctly; 2) the researcher's assurance that the patients used the inhaler device correctly; 3) error correction and reassessment by the researcher that the patients used the inhaler device properly; and 4) During the session, each patient was given a 'booklet' with photographs and instructions on how to correctly use an inhaler device in simple Arabic language to assist them to remember what they had learned.

Part II: Video education method: the researcher has presented this video which explains how to use an inhaler device correctly according to its type on the researcher's laptop. This video was developed by the pharmacy AIN shams university faculty containing comprehensive knowledge on how to use the inhaler device correctly. Every patient who has a cellphone can obtain a copy of the video. This session took about 10 minutes, after that there were 5 - 10 minutes for discussion and gave feedback

Part III: In this section, the researcher used a combination of a nursing educational booklet and a video teaching strategy about the correct use of inhaler device.

Ethical considerations:

Permission to conduct the study was obtained from the hospital authorities of chest Department at Assiut University Hospital. Prior to the initial interview, the researchers introduced themselves to patients who met the inclusion criteria; each potential patient was fully informed with the purpose and nature of the study, and then informed consent was obtained from participants who accept to participate in the study. The researchers emphasized that participation in the study is entirely voluntary and withdrawal from the study would not affect the care provided; anonymity and confidentiality were assured through coding the data.

Tool validity

Face validity was revised and checked by (5) experts from Medical-Surgical Nursing Staff and Medical staff at Assuit University. The experts revised the developed tools for clarity, relevance and comprehensive. Minor changes were done. Reliability was assessed by Cronbach's alpha $=0.90$. 


\section{Pilot study}

It was applied to $10 \%$ of the studied sample (8 patients) to assess the feasibility of the study and detect any difficulties needed to be handled. According to the results of this pilot study, the necessary changes were made.

Data collection: The data collection was done through the following phases:

Preparatory phase:

The researcher obtained an oral agreement from asthmatic patients for voluntary participation in the study.

The patients were divided into four groups, each with 20 individuals. To ensure a random distribution of patients, the odd numbers were assigned to the routine nursing instructions group and the group of patients who learned clinical correct use of inhaler through video only, while the even numbers were given to patients group who took the nursing educational booklet and those who learned through the video and the booklet for example, the first patient received routine nursing instructions, the second patient received nursing educational booklet, the third patient received clinical correct use of inhaler through video only, and the last received both educational booklet and video.

The researcher interviewed each patient individually to obtain the base line data that were established using patient assessment sheet (tool I part 1), evaluate the medical data of the patients were evaluated using ( tool I part 2), Risk factors for asthma exacerbations were evaluated using ( tool I part 3), and asthma complications were evaluated using( tool I part 4). Then the level of asthma symptoms control in the past 4 weeks was assessed using (Tool II).

\section{Implementation phase:}

The first group of 20 patients got routine nursing instructions (the researcher did not participate in educating patients on correct inhaler use and they considered as a control group).

The second group of patients received a nursing educational booklet (Tool III part I); each patient received the instructions in one session. The session takes 15 and 30 minutes. There was a 5-minute discussion and feedback period after each session. Teaching was reinforced based on the needs of the patients to ensure that they understood. Each patient was given a copy of the teaching booklet, which was illustrated with photographs and instructions in in simple Arabic language.

Patients in the third group were taught how to use the inhaler device correctly through video only on the researcher's laptop using (Tool III part II). Every patient who has a cellphone can obtain a copy of the video. This session took about 10 minutes, after that there were 5 - 10 minutes for discussion and gave feedback

The twenty patients of last group received combination of a nursing educational booklet and a video teaching strategy about the correct use of inhaler device by the laptop of the researcher using (Tool III part III). All patients in this group can obtain a copy from the booklet and video. After showing the video and explain the educational booklet, there were $5-10$ minutes for discussion and gave feedback. The collection of data lasted through the period from September 2020 to March 2021.

Evaluation phase:

After 3 months from the patient discharge from the hospital, the researcher met every patient in the four groups to evaluate the level of asthma control by using the Global Initiative for Asthma scale (GINA), 2019 ( tool II). This interview was conducted at the chest outpatient clinic

\section{Statistical analysis}

The data were tested for normality using the Anderson-Darling test and for homogeneity variances prior to further statistical analysis. Categorical variables were described by number and percent $(\mathrm{N}$, $\%)$, where continuous variables described by mean and standard deviation (Mean, SD). Chi-square test and fisher exact test used to compare between categorical variables where comparing between continuous variables was done by t-test and ANOVA, a two-tailed $\mathrm{p}<0.05$ was considered statistically significant. All analyses were performed with the IBM SPSS 20.0 software 


\section{Results:}

Table (I): Frequency distribution of demographic characteristic for study groups of patients with bronchial asthma

\begin{tabular}{|c|c|c|c|c|c|c|c|c|c|c|c|}
\hline \multirow[t]{2}{*}{$\begin{array}{l}\text { Demographic } \\
\text { characteristic }\end{array}$} & \multicolumn{2}{|c|}{$\begin{array}{c}\text { Routine } \\
\text { nursing care } \\
(n=20)\end{array}$} & \multicolumn{2}{|c|}{$\begin{array}{c}\text { Booklet } \\
(n=20)\end{array}$} & \multicolumn{2}{|c|}{$\begin{array}{c}\text { Video } \\
(n=20)\end{array}$} & \multicolumn{2}{|c|}{$\begin{array}{c}\text { Booklet } \\
\text { +video } \\
(n=20)\end{array}$} & \multicolumn{2}{|c|}{$\operatorname{Total}(n=80)$} & \multirow[t]{2}{*}{ P. value } \\
\hline & N. & $\%$ & N. & $\%$ & N. & $\%$ & N. & $\%$ & N. & $\%$ & \\
\hline \multicolumn{12}{|l|}{ Age group: } \\
\hline $20-<55$ years & 6 & 30.0 & 6 & 30.0 & 10 & 50.0 & 6 & 30.0 & 28 & 35.0 & \multirow{2}{*}{0.451} \\
\hline$\geq 55$ years & 14 & 70.0 & 14 & 70.0 & 10 & 50.0 & 14 & 70.0 & 52 & 65.0 & \\
\hline Mean \pm SD & \multicolumn{2}{|c|}{$49.2 \pm 11.91$} & \multicolumn{2}{|c|}{$52.6 \pm 13.31$} & \multicolumn{2}{|c|}{$47.35 \pm 8.31$} & \multicolumn{2}{|c|}{$44.5 \pm 12.18$} & \multicolumn{2}{|c|}{$48.41 \pm 11.74$} & 0.170 \\
\hline \multicolumn{12}{|l|}{ Gender: } \\
\hline Male & 8 & 40.0 & 8 & 40.0 & 2 & 10.0 & 6 & 30.0 & 24 & 30.0 & \multirow{2}{*}{0.126} \\
\hline Female & 12 & 60.0 & 12 & 60.0 & 18 & 90.0 & 14 & 70.0 & 56 & 70.0 & \\
\hline \multicolumn{12}{|l|}{ Marital Status: } \\
\hline Single & 2 & 10.0 & 0 & 0.0 & 0 & 0.0 & 0 & 0.0 & 2 & 2.5 & \multirow{3}{*}{0.080} \\
\hline Married & 18 & 90.0 & 16 & 80.0 & 19 & 95.0 & 18 & 90.0 & 71 & 88.8 & \\
\hline Divorced & 0 & 0.0 & 4 & 20.0 & 1 & 5.0 & 2 & 10.0 & 7 & 8.8 & \\
\hline \multicolumn{12}{|l|}{ Level of education: } \\
\hline High education & 2 & 10.0 & 0 & 0.0 & 1 & 5.0 & 0 & 0.0 & 3 & 3.8 & \multirow{5}{*}{0.079} \\
\hline Secondary education & 2 & 10.0 & 2 & 10.0 & 4 & 20.0 & 2 & 10.0 & 10 & 12.5 & \\
\hline Read and write & 8 & 40.0 & 4 & 20.0 & 5 & 25.0 & 10 & 50.0 & 27 & 33.8 & \\
\hline Illiterate & 0 & 0.0 & 6 & 30.0 & 4 & 20.0 & 0 & 0.0 & 10 & 12.5 & \\
\hline Primary education & 8 & 40.0 & 8 & 40.0 & 6 & 30.0 & 8 & 40.0 & 30 & 37.5 & \\
\hline \multicolumn{12}{|l|}{ Occupation: } \\
\hline Employee & 2 & 10.0 & 2 & 10.0 & 5 & 25.0 & 2 & 10.0 & 11 & 13.8 & \multirow{6}{*}{0.100} \\
\hline Farmer & 4 & 20.0 & 2 & 10.0 & 1 & 5.0 & 2 & 10.0 & 9 & 11.3 & \\
\hline House wife & 10 & 50.0 & 8 & 40.0 & 13 & 65.0 & 9 & 45.0 & 40 & 50.0 & \\
\hline Retired & 1 & 5.0 & 4 & 20.0 & 0 & 0.0 & 1 & 5.0 & 6 & 7.5 & \\
\hline Industrial worker & 2 & 10.0 & 0 & 0.0 & 0 & 0.0 & 4 & 20.0 & 6 & 7.5 & \\
\hline manual worker & 1 & 5.0 & 4 & 20.0 & 1 & 5.0 & 2 & 10.0 & 8 & 10.0 & \\
\hline \multicolumn{12}{|l|}{ Residence } \\
\hline Rural & 12 & 60.0 & 12 & 60.0 & 8 & 40.0 & 14 & 70.0 & 46 & 57.5 & \multirow{2}{*}{0.274} \\
\hline Urban & 8 & 40.0 & 8 & 40.0 & 12 & 60.0 & 6 & 30.0 & 34 & 42.5 & \\
\hline
\end{tabular}

- Chi square test for qualitative data between the two groups

Table (2): Frequency distribution of medical Data for study groups of patients with bronchial asthma

\begin{tabular}{|c|c|c|c|c|c|c|c|c|c|c|c|}
\hline & \multicolumn{2}{|c|}{$\begin{array}{c}\text { Routine } \\
\text { nursing care } \\
(\mathbf{n}=\mathbf{2 0})\end{array}$} & \multicolumn{2}{|c|}{$\begin{array}{c}\text { Booklet } \\
(n=20)\end{array}$} & \multicolumn{2}{|c|}{$\begin{array}{c}\text { Video } \\
(n=20)\end{array}$} & \multicolumn{2}{|c|}{$\begin{array}{c}\text { Booklet } \\
\text { +video } \\
(n=20)\end{array}$} & \multicolumn{2}{|c|}{$\begin{array}{c}\text { Total } \\
(\mathbf{n}=\mathbf{8 0})\end{array}$} & \multirow[t]{2}{*}{ P. value } \\
\hline & N. & $\%$ & $\mathbf{N}$. & $\%$ & $\mathbf{N}$. & $\%$ & N. & $\%$ & N. & $\%$ & \\
\hline $\begin{array}{l}\text { Previous admission } \\
\text { to hospital }\end{array}$ & 18 & 90.0 & 14 & 70.0 & 10 & 50.0 & 14 & 70.0 & 46 & 57.5 & $<0.001 * *$ \\
\hline \multicolumn{12}{|c|}{ Number of admission in the last year: } \\
\hline 1.00 & 0 & 0.0 & 0 & 0.0 & 0 & 0.0 & 4 & 20.0 & 4 & 8.7 & \multirow{3}{*}{$0.005 * *$} \\
\hline 2.00 & 12 & 60.0 & 8 & 40.0 & 0 & 0.0 & 10 & 50.0 & 30 & 65.2 & \\
\hline 3.00 & 6 & 30.0 & 6 & 30.0 & 0 & 0.0 & 0 & 0.0 & 12 & 26.1 & \\
\hline \multicolumn{12}{|c|}{ Age of bronchial asthma: } \\
\hline Less than 5 year & 4 & 20.0 & 2 & 10.0 & 8 & 40.0 & 4 & 20.0 & 18 & 22.5 & \multirow{3}{*}{0.260} \\
\hline from $5-10$ years & 2 & 10.0 & 4 & 20.0 & 4 & 20.0 & 2 & 10.0 & 12 & 15.0 & \\
\hline More than 10 years & 14 & 70.0 & 14 & 70.0 & 8 & 40.0 & 14 & 70.0 & 50 & 62.5 & \\
\hline \multicolumn{12}{|c|}{ Patients condition at discharge: } \\
\hline Improved & 20 & 100.0 & 20 & 100.0 & 20 & 100.0 & 20 & 100.0 & 80 & 100.0 & - \\
\hline \multicolumn{12}{|l|}{ Presence of cough } \\
\hline Present & 20 & 100.0 & 20 & 100.0 & 20 & 100.0 & 20 & 100.0 & 80 & 100.0 & - \\
\hline
\end{tabular}




\begin{tabular}{|c|c|c|c|c|c|c|c|c|c|c|c|}
\hline & \multicolumn{2}{|c|}{$\begin{array}{c}\text { Routine } \\
\text { nursing care } \\
(\mathbf{n}=\mathbf{2 0})\end{array}$} & \multicolumn{2}{|c|}{$\begin{array}{c}\text { Booklet } \\
(n=20)\end{array}$} & \multicolumn{2}{|c|}{$\begin{array}{c}\text { Video } \\
(n=20)\end{array}$} & \multicolumn{2}{|c|}{$\begin{array}{c}\text { Booklet } \\
\text { +video } \\
(\mathrm{n}=20) \\
\end{array}$} & \multicolumn{2}{|c|}{$\begin{array}{c}\text { Total } \\
(\mathbf{n}=\mathbf{8 0})\end{array}$} & \multirow[t]{2}{*}{ P. value } \\
\hline & N. & $\%$ & N. & $\%$ & N. & $\%$ & $\mathbf{N}$. & $\%$ & N. & $\%$ & \\
\hline \multicolumn{12}{|c|}{ If yes how much you cough } \\
\hline Sometimes & 4 & 20.0 & 8 & 40.0 & 6 & 30.0 & 6 & 30.0 & 24 & 30.0 & \multirow{4}{*}{$0.005 * *$} \\
\hline Moderate & 6 & 30.0 & 0 & 0.0 & 10 & 50.0 & 4 & 20.0 & 20 & 25.0 & \\
\hline Severe & 4 & 20.0 & 4 & 20.0 & 0 & 0.0 & 8 & 40.0 & 16 & 20.0 & \\
\hline Throughout the day & 6 & 30.0 & 8 & 40.0 & 4 & 20.0 & 2 & 10.0 & 20 & 25.0 & \\
\hline \multicolumn{12}{|l|}{ Shortness of breath: } \\
\hline Present & 20 & 100.0 & 20 & 100.0 & 20 & 100.0 & 20 & 100.0 & 80 & 100.0 & - \\
\hline \multicolumn{12}{|l|}{ Wheezing: } \\
\hline Present & 20 & 100.0 & 20 & 100.0 & 20 & 100.0 & 20 & 100.0 & 80 & 100.0 & - \\
\hline \multicolumn{12}{|l|}{ Chest tightness or pain: } \\
\hline Present & 20 & 100.0 & 20 & 100.0 & 20 & 100.0 & 20 & 100.0 & 80 & 100.0 & - \\
\hline \multicolumn{12}{|l|}{ Type of inhaler: } \\
\hline Dry powder inhaler & 10 & 50.0 & 8 & 40.0 & 12 & 60.0 & 14 & 70.0 & 44 & 55.0 & \multirow{2}{*}{$0.011 *$} \\
\hline Metered dose inhaler & 4 & 20.0 & 10 & 50.0 & 8 & 40.0 & 6 & 30.0 & 28 & 35.0 & \\
\hline $\begin{array}{l}\text { Dry powder inhaler } \\
\text { \&Metered dose inhaler }\end{array}$ & 6 & 30.0 & 2 & 10.0 & 0 & 0.0 & 0 & 0.0 & 8 & 10.0 & \\
\hline \multicolumn{12}{|l|}{ Chronic disease } \\
\hline Yes & 16 & 80.0 & 14 & 70.0 & 8 & 40.0 & 16 & 80.0 & 54 & 67.5 & \\
\hline \multicolumn{12}{|l|}{ If Yes } \\
\hline DM & 10 & 50.0 & 8 & 40.0 & 0 & 0.0 & 0 & 0.0 & 18 & 22.5 & $<0.001 * *$ \\
\hline Hypertension & 16 & 80.0 & 12 & 60.0 & 8 & 40.0 & 16 & 80.0 & 52 & 65.0 & $0.022 *$ \\
\hline Cardiovascular disease & 10 & 50.0 & 6 & 30.0 & 0 & 0.0 & 0 & 0.0 & 16 & 20.0 & $<0.001 * *$ \\
\hline Neurological disease & 0 & 0.0 & 0 & 0.0 & 0 & 0.0 & 0 & 0.0 & 0 & 0.0 & - \\
\hline DVT & 0 & 0.0 & 2 & 10.0 & 0 & 0.0 & 0 & 0.0 & 2 & 2.5 & 0.104 \\
\hline Tumors disease & 0 & 0.0 & 0 & 0.0 & 0 & 0.0 & 0 & 0.0 & 0 & 0.0 & - \\
\hline
\end{tabular}

\section{- Chi square test for qualitative data between the two groups}

* Significant level at P value $<0.05$,

** High significant level at P value $<0.01$

Table (3): Frequency distribution of risk factors for asthma exacerbation for study groups of patients.

\begin{tabular}{|c|c|c|c|c|c|c|c|c|c|c|c|}
\hline \multirow[t]{2}{*}{ Risk Factor } & \multicolumn{2}{|c|}{$\begin{array}{c}\text { Routine } \\
\text { nursing care } \\
(\mathbf{n}=\mathbf{2 0})\end{array}$} & \multicolumn{2}{|c|}{$\begin{array}{c}\text { Booklet } \\
(n=20)\end{array}$} & \multicolumn{2}{|c|}{$\begin{array}{c}\text { Video } \\
(\mathbf{n}=\mathbf{2 0})\end{array}$} & \multicolumn{2}{|c|}{$\begin{array}{c}\text { Booklet } \\
\text { +video } \\
(n=20)\end{array}$} & \multicolumn{2}{|c|}{$\begin{array}{c}\text { Total } \\
(\mathbf{n}=80)\end{array}$} & \multirow[t]{2}{*}{ P. value } \\
\hline & N. & $\%$ & N. & $\%$ & N. & $\%$ & $\mathbf{N}$. & $\%$ & N. & $\%$ & \\
\hline Uncontrolled asthma Symptoms & 20 & 100.0 & 8 & 40.0 & 18 & 90.0 & 20 & 100.0 & 66 & 82.5 & $<0.001 * *$ \\
\hline Medication & 0 & 0.0 & 0 & 0.0 & 0 & 0.0 & 2 & 10.0 & 2 & 2.5 & 0.104 \\
\hline $\begin{array}{l}\text { high SABA use if }>1 * 200 \text { dose } \\
\text { canister/ month }\end{array}$ & 0 & 0.0 & 0 & 0.0 & 0 & 0.0 & 2 & 10.0 & 2 & 2.5 & 0.104 \\
\hline inadequate ICS & 0 & 0.0 & 4 & 20.0 & 0 & 0.0 & 4 & 20.0 & 8 & 10.0 & $0.031 *$ \\
\hline Poor adherence & 10 & 50.0 & 14 & 70.0 & 10 & 50.0 & 6 & 30.0 & 40 & 50.0 & 0.094 \\
\hline Incorrect inhaler technique & 8 & 40.0 & 2 & 10.0 & 10 & 50.0 & 8 & 40.0 & 28 & 35.0 & $0.048^{*}$ \\
\hline \multicolumn{12}{|l|}{ Comorbidity } \\
\hline Obesity & 2 & 10.0 & 8 & 40.0 & 0 & 0.0 & 2 & 10.0 & 12 & 15.0 & \multirow{3}{*}{$<0.001 * *$} \\
\hline Chronic rhino Sinusitis & 18 & 90.0 & 12 & 60.0 & 16 & 80.0 & 18 & 90.0 & 64 & 80.0 & \\
\hline Obesity\&chronic rhino Sinusitis & 0 & 0.0 & 0 & 0.0 & 4 & 20.0 & 0 & 0.0 & 4 & 5.0 & \\
\hline \multicolumn{12}{|l|}{ Exposure } \\
\hline Smoking & 12 & 60.0 & 4 & 20.0 & 12 & 60.0 & 12 & 60.0 & 40 & 50.0 & \multirow{5}{*}{$<0.001 * *$} \\
\hline Allergen Exposure & 0 & 0.0 & 2 & 10.0 & 4 & 20.0 & 0 & 0.0 & 6 & 7.5 & \\
\hline Air pollution & 8 & 40.0 & 8 & 40.0 & 4 & 20.0 & 6 & 30.0 & 26 & 32.5 & \\
\hline Smoking \&Air pollution & 0 & 0.0 & 0 & 0.0 & 0 & 0.0 & 2 & 10.0 & 2 & 2.5 & \\
\hline Allergen Exposure \&Air pollution & 0 & 0.0 & 6 & 30.0 & 0 & 0.0 & 0 & 0.0 & 6 & 7.5 & \\
\hline
\end{tabular}




\begin{tabular}{|c|c|c|c|c|c|c|c|c|c|c|c|}
\hline \multirow[t]{2}{*}{ Risk Factor } & \multicolumn{2}{|c|}{$\begin{array}{c}\text { Routine } \\
\text { nursing care } \\
(\mathbf{n}=\mathbf{2 0})\end{array}$} & \multicolumn{2}{|c|}{$\begin{array}{c}\text { Booklet } \\
(\mathbf{n}=\mathbf{2 0})\end{array}$} & \multicolumn{2}{|c|}{$\begin{array}{c}\text { Video } \\
(\mathbf{n}=\mathbf{2 0})\end{array}$} & \multicolumn{2}{|c|}{$\begin{array}{l}\text { Booklet } \\
\text { +video } \\
(\mathbf{n}=\mathbf{2 0}) \\
\end{array}$} & \multicolumn{2}{|c|}{$\begin{array}{c}\text { Total } \\
(\mathbf{n}=\mathbf{8 0})\end{array}$} & \multirow[t]{2}{*}{ P. value } \\
\hline & N. & $\%$ & N. & $\%$ & N. & $\%$ & N. & $\%$ & N. & $\%$ & \\
\hline \multicolumn{12}{|l|}{ Context } \\
\hline Major psychological & 14 & 70.0 & 14 & 70.0 & 8 & 40.0 & 14 & 70.0 & 50 & 62.5 & \multirow{2}{*}{0.124} \\
\hline Socioeconomic Problem & 6 & 30.0 & 6 & 30.0 & 12 & 60.0 & 6 & 30.0 & 30 & 37.5 & \\
\hline
\end{tabular}

Chi square test for qualitative data between the two groups

*Significant level at $P$ value $<0.05$,

** High significant level at $P$ value $<0.01$

Table (4): Frequency distribution of complications for study groups of patients with bronchial asthma

\begin{tabular}{|c|c|c|c|c|c|c|c|c|c|c|c|}
\hline \multirow[t]{2}{*}{ Complications } & \multicolumn{2}{|c|}{$\begin{array}{c}\text { Routine } \\
\text { nursing care } \\
(\mathbf{n}=\mathbf{2 0})\end{array}$} & \multicolumn{2}{|c|}{$\begin{array}{c}\text { Booklet } \\
(\mathbf{n}=20)\end{array}$} & \multicolumn{2}{|c|}{$\begin{array}{c}\text { Video } \\
(\mathbf{n}=\mathbf{2 0})\end{array}$} & \multicolumn{2}{|c|}{$\begin{array}{c}\text { Booklet }+ \\
\text { video } \\
(\mathbf{n}=20)\end{array}$} & \multicolumn{2}{|c|}{$\begin{array}{c}\text { Total } \\
(\mathbf{n}=\mathbf{8 0})\end{array}$} & \multirow[t]{2}{*}{ P. value } \\
\hline & $\mathbf{N}$. & $\%$ & N. & $\%$ & N. & $\%$ & N. & $\%$ & N. & $\%$ & \\
\hline Pneumonia & 0 & 0.0 & 4 & 20.0 & 0 & 0.0 & 0 & 0.0 & 4 & 5.0 & $0.006 * *$ \\
\hline Respiratory infection & 0 & 0.0 & 0 & 0.0 & 0 & 0.0 & 0 & 0.0 & 0 & 0.0 & - \\
\hline Respiratory Failure & 0 & 0.0 & 0 & 0.0 & 0 & 0.0 & 0 & 0.0 & 0 & 0.0 & - \\
\hline Status asthmatics & 0 & 0.0 & 0 & 0.0 & 4 & 20.0 & 0 & 0.0 & 4 & 5.0 & $0.006 * *$ \\
\hline Asthma Exacerbation & $\overline{14}$ & 70.0 & 0 & 0.0 & 6 & 30.0 & 4 & 20.0 & 24 & 30.0 & $<0.001 * *$ \\
\hline Lack of Physical activity & 12 & 60.0 & 12 & 60.0 & 4 & 20.0 & 10 & 50.0 & 38 & 47.5 & $0.035^{*}$ \\
\hline Fatigue & 16 & 80.0 & 12 & 60.0 & 6 & 30.0 & 8 & 40.0 & 42 & 52.5 & $0.008 * *$ \\
\hline Sleep difficulties & 12 & 60.0 & 8 & 40.0 & 8 & 40.0 & 12 & 60.0 & 40 & 50.0 & 0.362 \\
\hline
\end{tabular}

- Chi square test for qualitative data between the two groups

- *Significant level at $P$ value $<0.05$, ** High significant level at $P$ value $<0.01$

Table (5): Frequency distribution of asthma Symptom control according to (GINA guidelines, 2019) for study groups of patients with bronchial asthma before and after education ( 3 months)

\begin{tabular}{|c|c|c|c|c|c|c|c|c|c|}
\hline \multirow[t]{2}{*}{ Level of Asthma Symptom control } & \multicolumn{2}{|c|}{$\begin{array}{c}\text { Routine } \\
\text { nursing care } \\
(\mathbf{n}=\mathbf{2 0})\end{array}$} & \multicolumn{2}{|c|}{$\begin{array}{c}\text { Booklet } \\
(n=20)\end{array}$} & \multicolumn{2}{|c|}{$\begin{array}{c}\text { Video } \\
(n=20)\end{array}$} & \multicolumn{2}{|c|}{$\begin{array}{c}\text { Booklet } \\
\text { +video } \\
(n=20)\end{array}$} & \multirow[t]{2}{*}{ P. value } \\
\hline & N. & $\%$ & N. & $\%$ & N. & $\%$ & N. & $\%$ & \\
\hline \multicolumn{9}{|l|}{ Before education } & \\
\hline Well Controlled & 0 & 0.0 & 0 & 0.0 & 0 & 0.0 & 0 & 0.0 & \multirow{3}{*}{$0.048^{*}$} \\
\hline Partly Controlled & 2 & 10.0 & 0 & 0.0 & 4 & 20.0 & 0 & 0.0 & \\
\hline Uncontrolled & 18 & 90.0 & 20 & 100.0 & 16 & 80.0 & 20 & 100.0 & \\
\hline Mean \pm SD & \multicolumn{2}{|c|}{$3.5 \pm 0.69$} & \multicolumn{2}{|c|}{$3.9 \pm 0.31$} & \multicolumn{2}{|c|}{$3.4 \pm 0.82$} & \multicolumn{2}{|c|}{$3.3 \pm 0.47$} & $0.014 *$ \\
\hline \multicolumn{10}{|l|}{ After education (3 months) } \\
\hline Well Controlled & 2 & 10.0 & 8 & 40.0 & 8 & 40.0 & 16 & 80.0 & \multirow{3}{*}{$<0.001 * *$} \\
\hline Partly Controlled & 12 & 60.0 & 12 & 60.0 & 12 & 60.0 & 4 & 20.0 & \\
\hline Uncontrolled & 6 & 30.0 & 0 & 0.0 & 0 & 0.0 & 0 & 0.0 & \\
\hline Mean \pm SD & \multicolumn{2}{|c|}{$1.8 \pm 1.01$} & \multicolumn{2}{|c|}{$0.8 \pm 0.77$} & \multicolumn{2}{|c|}{$0.9 \pm 0.85$} & \multicolumn{2}{|c|}{$0.4 \pm 0.82$} & $<0.001 * *$ \\
\hline
\end{tabular}

- Chi square test for qualitative data between the two groups

- One way Anova test quantitative data between the Three groups or more

*Significant level at $P$ value $<0.05$,

** High significant level at $\mathrm{P}$ value $<0.01$

Table (1): Illustrates that more over two-thirds of patients in the routine, booklet, and both booklet and video $(70 \%)$ are over fifty, while half of patients in the video $(50 \%)$ are over fifty. More than half of patients at routine, booklet, video, and both booklet and video were female. In terms of marital status, the majority of the patients in each of the four study groups were married. Regard to education, two fifth of routine nursing care, booklet, both booklet and video were primary education while more than one fifth of video group was primary education .more than two third of four study groups were housewives. More than half of the patients in the routine, booklet and both booklet and video groups lived in rural areas, whereas more than half of the video group 
lived in cities. No significant difference between four groups and Sociodemographic data.

Table (2): Shows that over two-thirds of patients in the routine, booklet, and both booklet and video groups had asthma for more than ten years, while two-fifths in the video group had asthma for less than 10 years. All patients in four groups complain of cough, shortness of breath, wheezing and chest tightness or pain. Majority of patients in all study groups suffered from hypertension. There are high significant difference between four groups and previous admission to hospital, Number of admission in the last year, presence of cough, DM, Cardiovascular diseases.

Table (3): Shows that majority of patients in four groups had uncontrolled asthma symptoms .Half and more patients in three groups (routine, booklet, video) had poor adherence and more than one quarter in both booklet and video group. Half and less in four groups had incorrect inhaler technique .more than half and majority of patients in four groups had chronic rhino sinusitis. More than half and one fifth of patients exposed to smoking. More than two thirds and one fifth of patients suffered from asthma symptoms through major psychological status. There are high significant difference between four groups and chronic rhino Sinusitis, smoking \&Air pollution

Table (4): Shows that majority of routine nursing care group had fatigue; over half of booklet group had lack of physical activity and fatigue. Two fifth of video group had sleep difficulties. More than half of both booklet and video had sleep difficulties. There are high significant difference between four groups and status asthmatics, asthma exacerbation, pneumonia.

Table (5): Shows that vast majority of patients in four groups had uncontrolled asthma symptoms before education .More than half of patients at routine, booklet and video had partly controlled asthma symptoms while majority of patients at both booklet and video had well controlled symptoms after education. There are high significant differences between four groups and asthma symptoms control after three months.

\section{Discussion:}

Patient education is a crucial part of asthma management. An assessment of a patient's knowledge of medicine and how they use it may aid in detecting issues in therapy and improving therapeutic outcomes. (Elbanna et al., 2017)

The usefulness of video education and video instructions are becoming progressively common ways of providing inhaler education. Interventions using a combination of educational videos, checklists, leaflets and verbal instructions found that this type of education significantly improved inhaler technique, additionally it was found to decrease attack frequency and dyspnea, and improved quality of life. (Schantz et al., 2018)

The aim of this study is to evaluate the effect of different nursing educational methods (video, educational booklet, video \& booklet and routine care) on clinical improvement for asthmatic patients.

Based on the result of the present study, that more than two-thirds of patients in routine, booklet, both booklet and video are over the age of fifty, whereas half of patients in video are over the age of fifty. More over half of the patients at routine, booklet, video, and both booklet and video were female. The results were in the same line with Fawzi, 2013; in a study named Assessing Appropriate Use of Inhaler Devices among Asthmatic Patients, the majority of the study participants were female.

In terms of marital status, the majority of the patients in each of the four study groups were married. This result disagreed with Paulo et al., 2014) who stated that Suspected asthma prevalence differed according to marital status, with single, divorced, and widowed adults having higher rates.

Regard to education, two fifth of routine nursing care, booklet, both booklet and video were primary education while more than one fifth of video group was primary education. The current study finding disagreed with Simon et al., 2009; who mentioned that more than half of the patients had a high level of education, and it was discovered that patients with lower levels of education had worse asthma control than those with higher levels of education.

Regarding occupation; more than two third of four study groups were housewives. This findings agreed with Taponen et al., 2018 showed that Having frequent asthma symptoms or nighttime wake-ups as a result of asthma is linked to less favourable employment outcomes, such as unemployment and work disability.

Regarding residence, more than half of patients at routine, booklet and both booklet and video were lived in rural areas while more than half of video group was lived in urban areas. This finding agreed with Beata et al., 2015 who revealed that; quite a large population of asthma patients lives in urban areas.

Also, Edard et al., 2014; reported that; Patients with asthma who live in inner city environment are at a higher risk. Certain ethnic groups within a population, such as Americans of African or Spanish inheritance may have a greater frequency of severe asthma.

The current study showed that half and more of four group patients admitted to hospital previously. More than two-thirds of patients in the routine, booklet, and both booklet and video groups had been diagnosed 
with asthma for more than ten years, while two-fifths of patients in the video group had been diagnosed with asthma for less than ten years. All patients in four groups complain of cough, shortness of breath, wheezing and chest tightness or pain. Majority of patients in all study groups suffered from hypertension.

Henriette et al., 2014; agreed with our study's findings and stated that Patients with numerous chronic illnesses had a threefold increased risk of any asthma-related hospital/ED admission, as well as a more than twofold increased risk of an unscheduled outpatient visit for urgent treatment of increasing asthma symptoms or an asthma attack.

Harpe et al., 2015; was also in the same line and mentioned that Comorbidities, such as chronic lung, psychiatric, and cardiovascular diseases are other risk factors.

The results of the current study revealed that the vast majority of patients in four groups had uncontrolled asthma symptoms. Half and more patients in three groups (routine, booklet, video) had poor adherence and in both the booklet and video groups, more than a quarter. Half and less in four groups had incorrect inhaler technique .more than half and majority of patients in four groups had chronic rhino sinusitis. More than half and one fifth of patients exposed to smoking. More than two thirds and one fifth of patients suffered from asthma symptoms through major psychological status.

From researcher opinion, poor adherence of patients is due to Limited access of the patient to appropriate health care and lack of education about appropriate management strategies.

Paulo et al., 2014; was agreed with the findings of the study and stated that incorrect inhaler technique in asthma treatment can significantly diminish medication deposition in the lungs, compromising asthma treatment effectiveness and being linked to poor asthma control, according to research. Smoking is another key element that influences the development of sickness in our patients.

Also Solet et al., 2019; was in the same line and reported that the relationship between asthma and environmental elements like moisture or cockroaches, as well as the usage of insecticide sprays at home

Ennifer et al., 2016 revealed that Poor adherence to prescribed treatments is a key risk factor for asthma that is both near fatal and lethal. Excessive use of b2agonists, concurrent use of b-blockers, and failure to prescribe or administer inhaled corticosteroids (ICSs) as a primary therapy are all examples of inadequate treatment. The patient's recent cessation of oral corticosteroids (OCSs) indicate that a severe exacerbation is more likely.
The current study's findings revealed that the majority of the routine nursing care group had fatigue, more than half of booklet group had lack of physical activity and fatigue. Two fifth of video group had sleep difficulties. More than half of both booklet and video had sleep difficulties.

Maarten et al., 2018 was agreed with the researcher's opinion and reported that about two-thirds of patients have significant exhaustion, and fatigue determines disease-specific QoL independently.

Sundbom , 2019 agreed with these findings which revealed that; In the Life Gene study, uncontrolled asthma was found to be a risk factor for all symptoms of insomnia. Sleep quality was negatively impacted by asthma-related comorbidity; in particular, the combination of uncontrolled asthma and any comorbidities was particularly unfavorable. Insomnia symptoms and uncontrolled asthma were both linked to chronic rhino sinusitis.

The present study illustrated that vast majority of patients in four groups had uncontrolled asthma symptoms before education. More than half of patients receive a booklet and a video as part of their regular care had partly controlled asthma symptoms while majority of patients at both booklet and video had well controlled symptoms after education.

Elbanna et al., 2017 was in keeping with the findings of the current study, which demonstrated that; the educational intervention resulted in considerable improvements in asthma knowledge and asthma control. .Asthma education provided by the family increase the level of asthma knowledge in adult patients with mild to moderate asthma.

Also, Beata et al., 2015; mentioned that education for both women and men. Understanding the basics of asthma and how to treat it has a positive impact on quality of life and asthma control. Educational strategies that try to promote patient awareness may not always result in improved lung function or the need for medical intervention. The use of interactive education can help improve asthma therapy.

Finally, it can be concluded that several nursing educational methods can be used to educate asthmatic patients assist the patient to manage the disease in terms of improving asthma patients' knowledge, controlling episodes of asthma, preventing acute and chronic complications.

Limitation of the study:

The majority of patients who received video did not have access to a laptop or smartphone, thus they only saw the video once on the researcher's laptop.

\section{Conclusion:}

The group of patients who received the video and the booklet had a statistically significant effect on improving the level of asthma symptoms control. 


\section{Recommendation:}

All patients with asthma should be provided with written and visual information about the correct use of inhalers to reduce complications arising from the incorrect use of inhalers.

\section{Refrences:}

- Beata Jankowska-Polańska, Justyna Pleśniak, Mariola Seń, Izabella Uchmanowicz, Joanna \& Rosińczuk, (2015): importance of education in bronchial asthma treatment- gender differences; 83: 341-347

- Chrystyn H, van der Palen J, Sharma R, Device errors in asthma \& COPD, (2017): systematic literature review and meta-analysis. NPJ Prim Care Respire Med.; 27(1):22.

- Crane MA, Jenkins CR, \& Goeman DP, (2014): Inhaler device technique can be improved in older adults through tailored education: findings from a randomized controlled trial. Prim Care Respir Med; 24: 14034.

- David B, Christine S, Charles R.H, Wassel M, Richenda Cope, Chantal B, \& Karl P(2019): interventions to Reduce Ageism Against Older Adults: A Systematic Review and Meta-Analysis, Am J Public Health109( 8) e1-e9.

- Edard A, Varraso R, Sanchez M, ClavelChapelon F, Zock J-P, \& Kauffmann F, (2014): Cleaning sprays, household help and asthma among elderly women.Respir ,.Acute asthma, prognosis, and treatment, Med.;108:171-80

- Elbanna, RM, Ashraf E. Sileem' B ahga, M.S , \& \& Ibrahem, G (2017): effect of bronchial asthma education program on asthma control among adults at Mansoura district, Volume 66, issues 4, 561-56

- Ennifer E. Fergeson, DO, Shiven S. Patel, \& Richard F. Lockey, (2016): Fundamentals of allergy and immunology, Elsevier Inc. on behalf of the American Academy of Allergy, Asthma \& Immunology, volume 139,No (2) ,438-447.

- Fawzi ,O.A (2013): Assessing Appropriate Use of Inhaler Devices among Asthmatic Patients, This Thesis is Submitted in Partial Fulfillment of the Requirements for the Degree of Master of Clinical Pharmacy, Faculty of Graduate Studies, An-Najah National University, Nablus, Palestine.Pp 1-4

- Giraud V, N.\& Roche N (2017): Misuse of corticosteroid metered-dose inhaler is associated with decreased asthma stability Eur Respir J, 19 (2) , pp. 246-251

- Global initiative for asthma (2020): Global Strategy for Asthma Management and Prevention for adults and children older than 5years. Pp. 10-40 www.ginasthma.org.

- Global initiative for asthma (2019): Global Strategy for Asthma Management and Prevention for adults and children older than 5 years .Pp. 16-30 www.ginasthma.org.

- Global asthma report (2018): Auckland, New Zealand: Global Asthma Network, Asthma affects 339million people, Pp.12-35 .www.globalasthmanetwork.org

- Guevara J.P , Wolf F.M., Grum C.M., \& Clark N.M. (2018): Effects of educational interventions for self-management of asthma in children and adolescents: systematic review and meta-analysis BMJ, 326 (7402) (2003), pp. 1308-1309

- Harpe RA, Bearman N, Thornton CR, Husk K, \& Osborne NJ. (2015): Indoor fungal diversity and asthma: a meta-analysis and systematic review of risk factors. JAllergy Clin Immunol.;135:110-22.

- Henriette Steppuhn, Ute Langen, Thomas Keil, \& Christa Scheidt-Nave (2014): Chronic disease co-morbidity of asthma and unscheduled asthma care among adults: results of the national telephone health interview survey German Health Update (GEDA) 2009 and 2010,Mar; 23(1): 22-29.

- Hanania NA, King MJ, Braman SS, Saltoun C, Wise RA, \& Enright $P$ (2011): Asthma in the elderly: current understanding and future research needs-a report of a National Institute on Aging (NIA) workshop. J Allergy Clin Immunol. P: 128(3 Suppl):S4-24.

- Lavorini F ISRN Allergy. (2013): Inhaler devices - from theory to practice. Sanchis J, Corrigan C, Levy ML, Viejo JL, ADMIT Group Respir Med. 2013 Apr; 107(4):495-502.

- Lee SM, Chang YS, Kim CW, Kim TB, Kim SH, Kwon YE, Lee JM, Lee SK, Jeong JW, Park JW, Cho SH, Moon HB, Jee YK Allergy Asthma Immunol Res.( 2011): Skills in handling turbuhaler, diskus, and pressurized metered-dose inhaler in korean asthmatic patients. Jan; 3(1):46-52

- Maarten Van Herck Martijn A. Spruit, Chris Burtin, Remco Djamin, Jeanine Antons, Yvonne M. J. Goërtz, Zjala Ebadi, Daisy J. Janssen, Jan H. Vercoulen, Jeannette B. Peters, Melissa S. Y. Thong, Jacqueline Otker, Arnold Coors, Mirjam A. Sprangers, Jean W. Muris, Emiel F. Wouters, Alex J. \& van t Hul '(2018) : Fatigue is Highly Prevalent in Patients with Asthma and Contributes to the Burden of Disease, Dec; 7(12): 471.

- Miguel R.R, Esther M, Gacia-prado M, Janwillem K, \& Thys Vann D M, (2019): Wrong inhalation technique is associated to poor asthma clinical outcomes. Is there room for improvement, Pub med J; 25(1):18-26.

- Paulo de Tarso Roth Dalcin, Denis Maltz Grutcki, Paola Paganella Laporte, Paula Borges de Lima, Samuel Millán Menegotto, Rosemary \& Petrik Pereira (2014): Factors related to the incorrect use of inhalers by asthma patients, J Bras 
Pneumol. Factors related to the incorrect use of inhalers by asthma patients, J Bras Pneumol. 2014; 40(1):13-20

- Pharmacy AIN shams university faculty (2019): containing detailed information on how to use the inhaler device

- Schantz S., Katajavouri N, \& Juppo A., (2018): The Use of Video Instructions in Patient Education Promoting Correct Technique for Dry Powder Inhalers: An Investigation on Inhaler-Naïve Individuals, Pharmacy, 6(4), 106; https://doi.org/10.3390/pharmacy6040106

- Simon L Bacon, Anne Bouchard, Eric B Loucks \& Kim L Lavoie (2009): Individual-level socioeconomic status is associated with worse asthma morbidity in patients with asthma, Respiratory Research volume 10, Article number: 125

- Solet, C. Raherison-Semjen, E. Mariotti, Y. Le Strat, A. Gallay, E. Bertrand, N. Jahaly \& Filleu Soletet, L. (2019): A cross sectional survey to estimate prevalence and associated factors of asthma on Reunion Island, Indian. BMC Public Health 19:663 https://doi.org/10.1186/s12889-0197031-7

- Sundbom, F. (2019): Asthma and Sleep Disturbances. Associations to Comorbidities and Asthma Control. Digital Comprehensive Summaries of Uppsala Dissertations from the Faculty of Medicine 1552. 78 pp. Uppsala: Acta Universitatis Upsaliensis. ISBN 978-91-513-0596-7.

- Taponen, S, Lauri Lehtimäki, Kirsi Karvala,Ritva Luukkonen \& Jukka Uitti, taponen (2018):. Employment status and changes in working career in relation to asthma: a crosssectional survey, Journal of Occupational Medicine and Toxicology 13:8 https://doi.org/10.1186/s12995-018-0189-

- Tarraf, H., Aydin, O., Mungan D., Albader, M., Mahbouo, B, Dodle A., Lahlou A., Tariq L., Aziz F., \& El Hasnaoui, A., (2018): Prevalence of asthma among the adult general population of live middle eastern https://www.medicalnewstoday.com/articles/32566 6.php

- World health organization, ( 2019): global burden of 369 diseases and injuries in 204 countries and territories, 1990-2019:, a systematic analysis for the Global Burden of Disease Study .;396(10258):120422 